\title{
Ground-based \& WFPC2 Imaging of Fornax: Spatial Variations in Star Formation History
}

\author{
Eva K. Grebel \\ UCO/Lick Obs., University of California, Santa Cruz, CA 95064, USA \\ Peter B. Stetson \\ National Research Council, Herzberg Institute of Astrophysics, \\ Dominion Astrophysical Observatory, 5071 West Saanich Road, \\ Victoria, British Columbia, V8X 4M6, Canada
}

\begin{abstract}
Fornax shows a radial age gradient. The old population is the most extended one similar to what was found in other dwarf galaxies, while younger populations are more centrally concentrated. With $\approx-1.2$ dex the dominant intermediate-age population is much more metal-rich than the oldest populations traced by Fornax's globular clusters $(\approx-2$ dex, $15-11 \mathrm{Gyr}$ ). Star formation appears to have proceeded continuously with decreasing rates rather than in distinct episodes. Fornax contains the youngest population ever found in a dwarf spheroidal galaxy, 100-200 Myr, which makes its apparent lack of gas even more puzzling.
\end{abstract}

\section{Introduction}

The Fornax dwarf spheroidal galaxy (dSph) is the second most massive dSph in the Local Group (LG) and a nearby $(\sim 120 \mathrm{kpc})$ Milky Way companion. We present results from archival WFPC2 images centered on Fornax's globular clusters and a homogeneous $35^{\circ} \times 35^{\circ}$ mosaic of ground-based $B, R$ photometry (Stetson et al. 1998) obtained at the 1.5-m telescope at CTIO.

\section{WFPC2 data for Fornax's globular clusters and surrounding field population}

Four out of five globular clusters (GCs) in Fornax are as old as the oldest GCs in the Milky Way (Buonanno et al. 1998). Other galaxies with cluster formation during this oldest star formation epoch are the LMC and Sagittarius (e.g., Sarajedini et al. 1998). Fornax's GC\#4 near the center of this galaxy (Fig. 1) is several Gyr younger but also metal-poor (Marconi et al. 1999), while the most metal-poor clusters (GC\#1 and \#5, $\approx-2.2$ dex) are farthest from the center.

The surrounding field population around GC\#1,2,3, and 5 appears to be in part as old as the GCs. Near GC\#2 and 3 we find indications for up to $\approx 4$ - 5 Gyr younger subgiant branches and a sparsely populated intermediate-age red clump. 


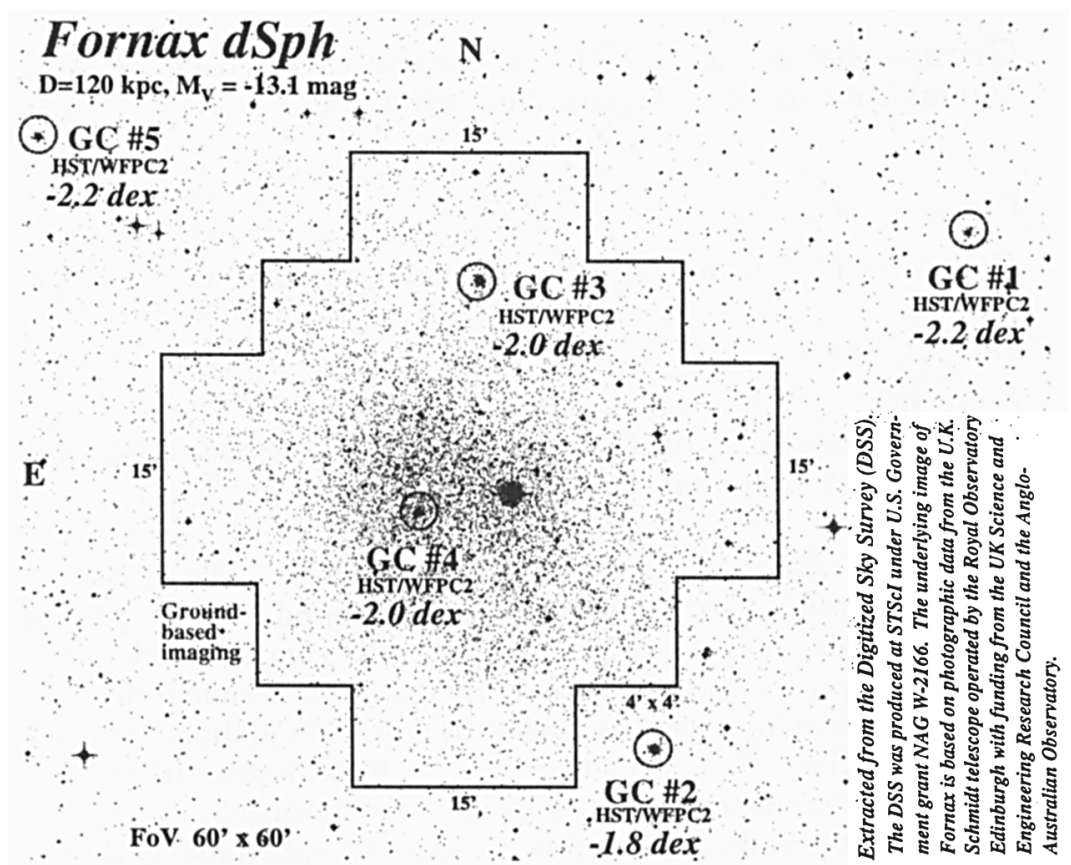

Figure 1. Image of Fornax from the Digitized Sky Survey. Fornax's five globular clusters (with mean metallicities from Buonanno et al. 1998 and Marconi et al. 1999) are marked by circles. Ground-based $B, R$ imaging was obtained for the central area enclosed by solid lines. The dominant intermediate-age field population has a mean metallicity of $-1.3 \mathrm{dex}$, much higher than the globular clusters.

\section{Fornax's central field population from ground-based imaging}

The ground-based color-magnitude diagrams (CMDs) show a mix of field populations. GC fiducials of Sarajedini \& Layden (1997) indicate a mean metallicity of $\approx-1.2$ dex. Near the center of Fornax the color distribution of red giants corresponds to a metallicity range of $\approx-1.4$ to $-0.7 \mathrm{dex}$, while the spread appears to decrease at the outskirts of the surveyed area (Fig. 2). Stars bluer than the -1.5 dex fiducial may be largely asymptotic giant branch (AGB) stars and younger red giants as indicated by the Bertelli isochrones. We cannot at present resolve the age-metallicity degeneracy along the red giant branch (RGB).

Near the galaxy center a wide, prominent red clump with a bright vertical extension (VRC) is seen, indicative of a substantial age spread among the intermediate-age population. The red clump is much more compact and less well populated at larger galactocentric distances (Fig. 2). Fig. 3 shows that the younger VRC population is more strongly centrally concentrated than the red clump population. Following Beaulieu \& Sackett (1998), we estimate an age of 


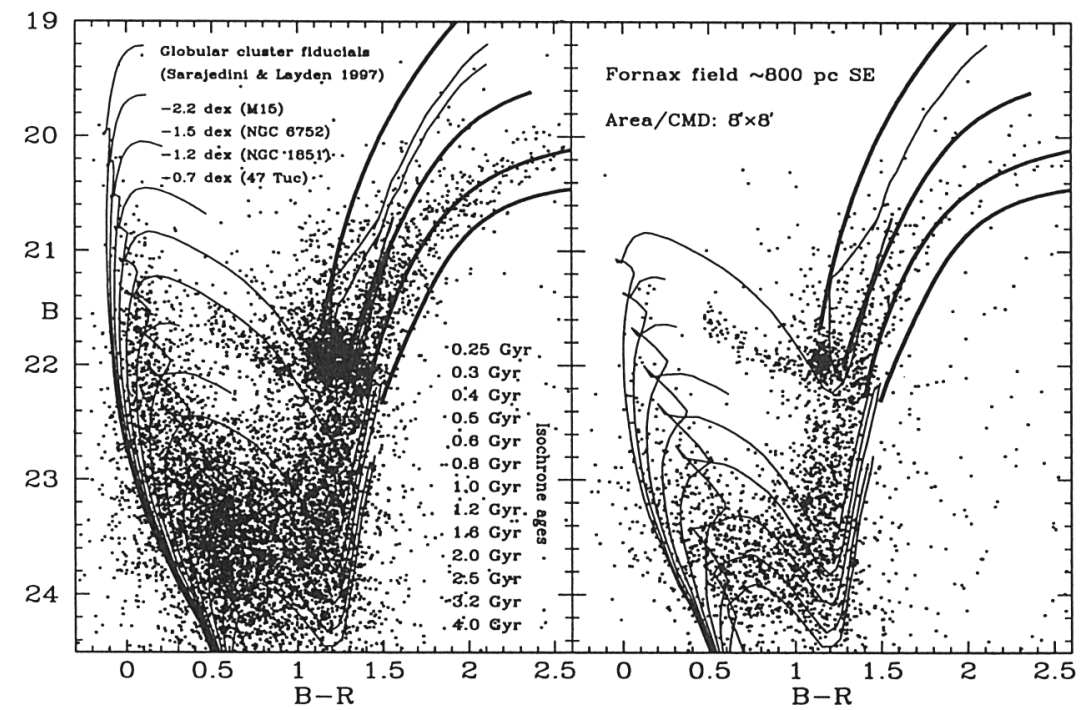

Figure 2. Color-magnitude diagrams for regions near the center of Fornax (left) and near the southeastern border of the surveyed area (right). The thin solid lines are isochrones (truncated to avoid obscuring the data points) from Bertelli et al. (1994). Bold lines on top of the RGB are globular cluster fiducials from Sarajedini \& Layden (1997).

$500 \mathrm{Myr}$ for the youngest VRC stars. Padua isochrones trace subgiants to ages as young as $\approx 0.6 \mathrm{Gyr}$, while star formation seems to have largely ended $1.6-2 \mathrm{Gyr}$ ago in the outskirts of the surveyed area. The main sequence in Fornax reaches ages as young as $\approx 200 \mathrm{Myr}$ (center and east (Fig. 3), Stetson et al. 1998). This is the youngest population ever found in a dSph galaxy, all the more remarkable considering the apparent lack of gas in Fornax. The magnitude range of the AGB bump (Alves \& Sarajedini 1999) near the center implies older intermediate-age populations $(5 \pm 2 \mathrm{Myr})$, which is corroborated by the isochrones. The data do not reach faint enough to include main-sequence/subgiant stars older than 4-5 Gyr.

The horizontal branch remains well-populated even at larger radii (see also the distribution of RR Lyrae stars in Fig. 3; the decrease at the edges of the surveyed area is an artifact of poorer time series sampling). The old field population appears to be rather evenly distributed throughout the galaxy as also indicated in the WFPC2 images.

A radial age and metallicity gradient with underlying, extended old populations is observed in a number of dwarf galaxies (see Grebel 1999 for a review). The available data suggest that Fornax showed continuous star formation with a decreasing star formation rate rather than episodic star formation as observed in Carina.

Acknowledgements. Support by D. Zaritsky through NASA LTSA grant NAG-5-3501 is gratefully acknowledged. 


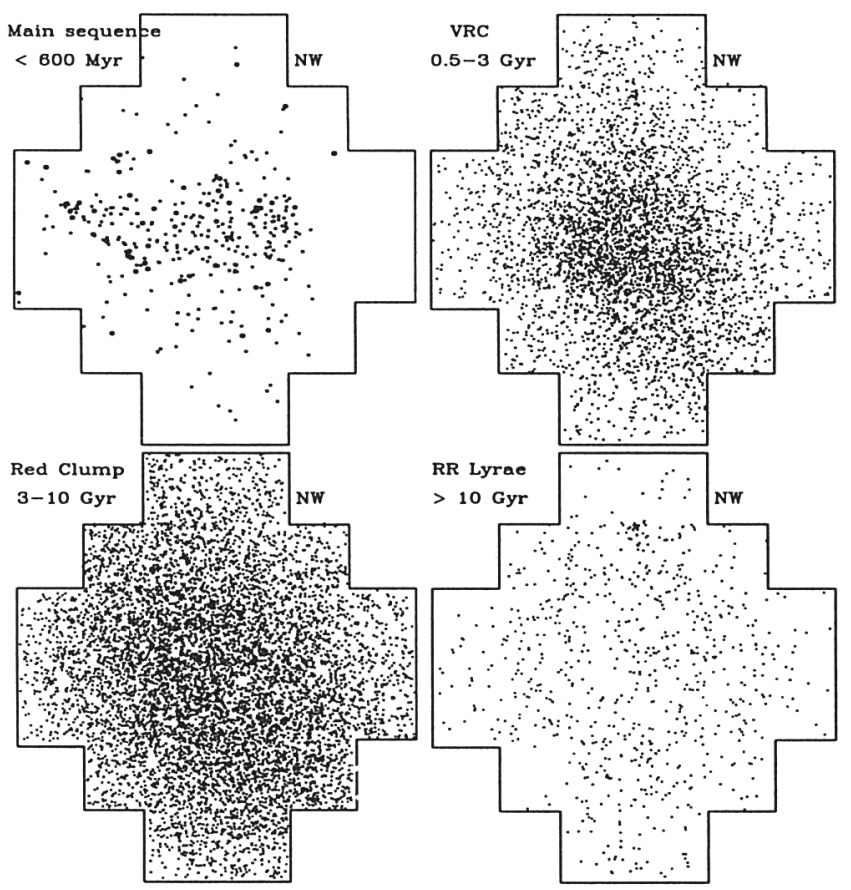

Figure 3. Distribution of stars of different ages in Fornax for the central area marked in Fig. 1. The younger populations are strongly centrally concentrated while the older ones are more evenly distributed.

\section{References}

Alves, D.R., Sarajedini, A. 1999, ApJ, in press (astro-ph/9808253)

Beaulieu, J.-P., Sackett, P.D. 1998, AJ, 116, 209

Bertelli, G., Bressan, A., Chiosi, C., Fagotto, F., Nasi, E. 1994, A\&AS, 106, 275

Buonanno, R., Corsi, C.E., Zinn, R., Fusi Pecci, F., Hardy, E., Suntzeff, N.B. 1998, ApJ, 501, L33

Grebel, E.K. 1999, in: The Stellar Content of the Local Group, IAU Symp. 192, (eds.) P. Whitelock \& R. Cannon, (San Francisco: ASP Conf. Ser.), in press

Marconi, G., Buonanno, R., Corsi, C.E., Zinn, R. 1999, in: The Stellar Content of the Local Group, IAU Symp. 192, (eds.) P. Whitelock \& R. Cannon, (San Francisco: ASP Conf. Ser.), in press

Sarajedini, A., Layden, A. 1997, AJ, 113, 264

Sarajedini, A., Geisler, D., Harding, P., Schommer, R. 1998, ApJ, 508, L37

Stetson, P.B., Hesser, J.E., Smecker-Hane, T.A. 1998, PASP, 110, 533 


\section{Discussion}

Feast: Can there be any effect of incompleteness in the distribution of RR Lyraes in Fornax which you showed?

Grebel: Yes. The central area of the surveyed region was observed more often and better sampled than the outer areas. The slight decrease in RR Lyr star density seen toward the outer areas may be caused in part by this incompleteness. This may imply that the distribution of the old population is very homogeneous and even. Ideally one would hope to have full-area coverage with well-sampled light curves to trace the distribution of the old population with a high degree of completeness. 